Voix et Images

voixetimages

\title{
Julien Bigras : l'Enfant dans le grenier
}

\section{André Brochu}

Volume 2, numéro 1, septembre 1976

Fernand Leduc

URI : https://id.erudit.org/iderudit/200026ar

DOI : https://doi.org/10.7202/200026ar

Aller au sommaire du numéro

\section{Éditeur(s)}

Les Presses de l'Université du Québec

\section{ISSN}

0318-9201 (imprimé)

1705-933X (numérique)

Découvrir la revue

\section{Citer cet article}

Brochu, A. (1976). Julien Bigras : l'Enfant dans le grenier. Voix et Images, 2(1), 119-123. https://doi.org/10.7202/200026ar d'utilisation que vous pouvez consulter en ligne.

https://apropos.erudit.org/fr/usagers/politique-dutilisation/ 


\section{Julien Bigras: l'Enfant dans le grenier}

II y a, dans les beaux-arts, une enclave réservée à la «peinture naïve». Marie Laurencin, le Douanier Rousseau; au Québec, Arthur Villeneuve, Gécin, les sceurs Bolduc ont illustré ce «genre», qui n'en est pas un. Si je comprends bien, pour être peintre naïf, il faut découvrir sur le tard la vocation de manier les pinceaux; il faut avoir vécu, comme seul un douanier ou un "barbier» peut le faire, ce qu'on appelle vivre (cf. le Grand Roman d'un petit homme, d'Yves Thériault); il faut enfin dessiner (c'est-à-dire représenter) comme un enfant. Vous avez quarante ou cinquante ans, vous êtes plongé depuis toujours et jusqu'au cou dans la quotidienneté, cependant un rêve vous habite, une nostalgie vous ronge et un jour, par miracle c'est-à-dire par hasard, vous vous mettez à peindre comme lorsque vous aviez dix ans, avec fraîcheur et maladresse, avec le sûr instinct de ces équilibres de forme et de couleur qui ne s'apprennent pas mais qui sont la peinture même, un monsieur de la ville vous découvre et c'est fait. Vous ne créerez aucune école mais vous apporterez, à la pratique générale de la chose picturale, une touche, un accent qui lui manquaient, la peinture peut être faite par tous et vous êtes l'élu qui le prouve.

Je ne connais pas, en littérature, l'équivalent exact. Par myopie, peut-être: la critique universitaire ne prédispose guère à ce genre de découverte. Victor-Lévy Beaulieu a publié un Manuel de la petite littérature du Québec dont les textes, tous maladroits, ont fait la délectation du très habile Jacques Ferron. Oui, il y a peut-être une littérature naïve, susceptible, avec le recul, de procurer l'enchantement. Et c'est un peu à cette littérature ou à la possibilité qu'elle représente - possibilité d'une certaine authenticité brute, aussi bien servie que compromise par l'ingénuité de la forme - que je pense à propos de l'Enfant dans le grenier'.

Julien Bigras n'est ni coiffeur ni douanier, mais sa profession de psychanalyste n'a pas fait de lui, pour autant que je sache, un «intellectuel ». Fondateur et directeur de la revue Interprétation, auteur d'un bel ouvrage sur les Images de la mère, il appartient à ce petit nombre d'individus - dont est, par exemple, Gaston Miron - qui, loin de se conformer à l'«image» de la fonction sociale qu'ils assument, la crèvent de toute part 
comme un vêtement trop serré. Ce qui ne les empêche pas d'être, qui poète, qui psychanalyste: mais ils le sont en étant aussi tout autre chose, et en particulier en étant eux-mêmes.

Je veux dire: en étant ce peuple dont ils proviennent et qui, en eux et par eux, cherche sa voie.

Avec une redoutable honnêteté.

En général, quand un savant s'adonne à la littérature, il écrit quelque chose comme Sonnets pour Noël et le carnaval (Pierre Demers), œuvrette sans prétention qu'on n'a pas même le goût de reprocher à son auteur. $\mathrm{Ce}$ qui caractérise ce genre de textes, c'est leur parfaite marginalité. Aux champs, le sous-préfet n'écrit pas, il "fait des vers" et c'est tout. Rien, de lui, ne passe dans les mots.

Julien Bigras, psychanalyste, ne pratique pas la littérature comme un passe-temps. II ne cherche pas à y oublier ce qu'il est: au contraire. II faut, ensemble, beaucoup de naïveté (au sens exact du terme) et de courage et d'authenticité pour, étant psychanalyste, se lancer dans un récit qui n'exclut pas, qui met en mots la situation psychanalytique. "Le divan sur lequel Joseph est étendu n'est pas à lui. Et ce bureau n'est pas le sien. C'est celui du docteur Douglevine. Pourtant Joseph se sent ici un peu comme s'il était chez lui. II raconte des histoires. Aujourd'hui c'est le souvenir de Frank qui lui vient à l'esprit. "Quel scandale, ce début de «roman»! Un psychanalyste, je le répète, qui vous flanque tout de suite le fameux divan par la tête et qui prétend pourtant faire de la «littérature»! Si grand, ce scandale, qu'on n'en croit pas ses yeux: ce divan, il a l'air d'un cliché, on n'imagine pas que la scène puisse être réelle tant il y $a$, en elle, d'attendu. Et pourtant...

Pour se conformer à nos habitudes de lecture, il aurait fallu, sans doute, que Julien Bigras se fasse d'abord écrivain, c'est-à-dire personne. - Nobody - ou persona... - Le récit, à ce moment, eût gagné en autonomie. Ici, il reste le fait d'un non-écrivain, c'est-à-dire, en fait, de n'importe qui. Voilà bien en quoi il s'agit de littérature naïve. "La poésie doit être faite par tous, écrivait Lautréamont. Non par un. Pauvre Hugo! Pauvre Racine! Pauvre Coppée! Pauvre Corneille! Pauvre Boileau! Pauvre Scarron! Tics, tics et tics. »

Il se peut, en effet, que la littérature ne doive plus être le privilège de quelques-uns. Des seuls gens du «métier».

Au cours de son histoire, la littérature a subi périodiquement des remises en question qui lui ont permis de se renouveler. II semble, cependant, qu'elle ait rarement connu crise plus grave que depuis une dizaine d'années. Cette crise en reflète une autre, plus générale, qui est celle des valeurs dans tout l'Occident capitaliste. Est-ce l'apparition de forces nouvelles de production, telles que l'ordinateur et certains media comme la télévișion, qui chambarde ainsi les rapports sociaux existants et crée un divorce d'une gravité sans précédent entre les modes de pensée reçus (idéo- 
logies) et l'infrastructure? Seule une analyse marxiste accordée aux réalités contemporaines pourrait nous éclairer là-dessus. Une mutation de la conscience contemporaine est en cours, qui n'a pas encore trouvé son expression révolutionnaire c'est-à-dire politique adéquate. II se peut aussi que le marxisme ne puisse plus, à lui seul, rendre compte des contradictions du monde actuel. Jacques Derrida a soutenu que la pensée actuelle logeait dans cette marge paradoxale qui se situe entre la clôture du logos platonicien et sa fin: qu'on ne peut plus ajouter aux divers systèmes de pensée qui se sont succédé dans le sillage de la problématique, instauratrice, de Platon; qu'on peut tout au plus, pour l'instant, déconstruire les fondements de la métaphysique occidentale et en rêver la fin, sans qu'il soit possible par ailleurs de tabler sur l'éventualité d'une autre histoire.

Dans le domaine des sciences humaines, au terme d'une époque qui en fut une de surspécialisation et de différenciation à l'extrême des problématiques particulières, on semble en arriver à un point de saturation et à la nécessité d'un rebrassage, d'une redistribution totale des faits de savoir. À l'intérieur de chaque discipline, le progrès ne semble plus possible qu'au prix d'un éclatement et d'une ouverture à l'interrogation de disciplines autres, ce qui entraîne fatalement pour elles une crise de l'objet. D'autre part, des disciplines syncrétiques, aux frontières mouvantes, vont se multipliant: sémiotique, communications..., dont l'existence et le destin semblent obéir étroitement aux caprices de l'instant (cf. feu la symbolique). Le champ du savoir se fractionne en toute son étendue, et les conditions d'une stabilisation n'existent guère pour le moment. Faudra-t-il, comme à la fin.du $X V I I I^{e}$ siècle, traverser une longue série de catastrophes pour que soit à nouveau possible un certain consensus autour de valeurs et de modèles socio-culturels? La littérature romantique, en même temps que s'instaurait une nouvelle épistémè (cf. M. Foucault), a proposé aux sociétés européennes une nouvelle foi en l'avenir de l'homme, et des mythologies appropriées à cet espoir.

L'entreprise "littéraire" de Julien Bigras me semble s'inscrire dans le contexte même que je viens d'évoquer. Psychanalyste, il lui est apparu que les moyens traditionnels de la psychanalyse ne lui suffisaient plus; que, derrière l'objet de l'analyse, se profilait un donné autre qui fonctionne, par rapport à celui-là, comme son «inconscient» et qui est peut-être, justement, l'inconscient lui-même, c'est-à-dire cela qui, aux racines du vivant, échappe à l'emprise du langage. Cet inconscient majeur ne devient perceptible que par la subversion de l'analyse, et c'est l'écriture narrative, "littéraire " qui opère ici cette subversion.

Les fondements "théoriques" de la démarche de Bigras reposent sur l'intuition d'une analogie, voire même d'une continuité entre le travail du 
rêve et le travail de l'écriture. Certes, la prudence est de rigueur quand on aborde une question si complexe, et il est bien évident qu'on ne saurait décréter une équivalence pure et simple entre l'onirique et le littéraire. Pas plus, par exemple, qu'entre le discours psychotique - le délire - et le discours poétique. Cependant, rien n'exclut que la littérature ne puisse, à la limite, coïncider (ou presque) avec telle ou telle autre pratique du langage: et, dans l'époque de crise où nous sommes, la littérature s'affirme beaucoup plus par ses expériences limites (songeons à la consécration de Sade, Mallarmé, Lautréamont, Artaud, Bataille) que par les autres.

Les limites de la littérature peuvent être de forme ou de contenu.

Le travail de Julien Bigras se situe principalement au plan du contenu. Il confie à l'écriture le soin de traquer une image, qui est un vécu. La forme n'a qu'à suivre. L' "artifice" littéraire, le code narratif sont subordonnés aux exigences d'une vérité - laquelle, cependant, est littéraire. Littéraire et vécue, indissociablement. l'écriture.

Littéraire, parce qu'appréhendée essentiellement par le moyen de

Et l'écriture ouvre ce vécu, qui est individuel, à l'espace-sens des significations collectives. La quête d'un personnage (Joseph), lui-même créateur de personnages (Karlouk), se révèle être celle du narrateur luimême (Joseph encore) et, finalement, de tous ces prénommés Joseph que nous sommes, nous les fils d'un Québec catholique et matriciel, ou matriarcal, que hantent justement les «images de la mère ${ }^{2}$ ". Joseph (le «Charpentier ) c'est l'Adam catholique, avec cependant une allure de prince consort qui laisse à la déesse-mère tous les attributs de la puissance. Fait cocu par une colombe, il ne peut rêver un messie qui soit issu de lui. Et nous, ses fils de même nom, portons tous en notre intime nuit la trace raturée d'un enfant foudroyé - d'un messie avorté.

Telle est l'une des résonances signifiantes possibles de ce qui nous est raconté. Mais il serait vain de chercher à contraindre en des formules univoques ce que l'auteur entend précisément laisser en état de silence, Jui qui cherche précisément, et non sans difficulté, à museler l'analyse et à nous conduire au-delà : à ce qui ne parle plus mais se parle - et se tait, tout à la fois.

En plus de la quête précise de soi à laquelle nous fait assister l'auteur, quête qui nous conduit, par une suite de révélations de plus en plus décisives, vers une connaissance et une acceptation de soi - comme dans une cure qui ne serait pas «interminable»... - , c'est au rare spectacle d'un certain passage que nous fait assister le texte de Bigras. Et ce passage est celui même du non-littéraire au littéraire.

Revenons, en effet, au début du texte: à ces courts chapitres qui nous présentent, entre guillemets, des souvenirs d'enfance. Tout, ici, nous parle de l'enfance, à commencer par le style lui-même, totalement dépourvu des marques de l'écriture au sens où l'entend Barthes, c'est-à-dire de ces signes par lesquels une œuvre marque son appartenance à la litté- 
rature, à une école. C'est qu'il manque encore, chez l'auteur, cette identification fondamentale à la fonction d'écrivain par laquelle se constitue comme telle l'écriture. On pourrait qualifier d'infantile ce stade de la pratique du langage où se manifeste une originalité par défaut, où la conduite du récit reste en deçà de l'imitation, laquelle est une étape indispensable pour l'instauration d'une visée spécifiquement littéraire.

La «deuxième partie» justement fait apparaître ce que j'appellerais l'œdipianisation de l'écriture et la référence à quelques grandes autorités textuelles: Joseph est mis en relation avec deux signifiants textuels chargés de connotations littéraires: le château et le procès (Kafka); avec «La douce" aussi, dont le nom rappelle sans peine Dostoïevsky. Cette extravagante histoire de meurtre, de procès dans un château, suivi de la survenue de la mère, nous plonge en pleine fiction - en plein mensonge littéraire - et ceci alors que Joseph nous est présenté dans son âge adulte, à distance de cette enfance «réelle " où le reportaient, précédemment, ses souvenirs.

Et c'est, dans un troisième temps, la fusion opérée entre le vrai et le faux, l'enfance ressouvenue et le présent de rêve; c'est la remontée vers le cauchemar initial, l'enfant du grenier dont la mort enténèbre le présent mais l'éclaire aussi, par la prise de conscience libératrice que fait Joseph du grand événement oublié. L'écriture acquiert alors, entre les eaux du vrai et du mensonge, le style de sa navigation propre: une aisance, une grâce qui sont l'indice sûr de la maîtrise assurée sur le vécu et, indissociablement, sur la visée littéraire elle-même. Ce que nous démontre ici Bigras, à nous littéraires qui l'avions oublié, c'est que la parole juste, sans parade, prise dans l'écheveau d'un réel aussi irrécusable que problématique, est le moteur véritable du texte beau; que la ferveur, quand elle triomphe de la (de sa) maladresse, en fait le terreau des éclosions du merveilleux, aux frontières du "vivre" et du "livre" (Meschonnic), du rêve et de l'écriture.

Ce qui me plaît enfin, c'est l'absence de décalage entre la façon d'être, de dire de l'auteur et celle de son peuple resté près des choses simples, mal adapté aux ratiocinations débridées propres à notre "ère exponentielle». La voilà, sans doute, en sa vérité profonde, la naïveté dont j'ai parlé: accord, au plus près, de la pensée avec le geste, et la chair, et la voix.

A cette fraîcheur, notre littérature a certes besoin de se ressourcer.

André Brochu

1. Julien Bigras, l'Enfant dans le grenier, Montréal, Parti pris, 1976.

2. Cf. Julien Bigras, les Images de la mère, Montréal et Paris, Interprétation et Hachette, 1971. 